\title{
63 SINGAPORE NURSING STUDENTS' PERCEPTIONS ABOUT SPIRITUALITY AND SPIRITUAL CARE: A QUALITATIVE STUDY
}

10.1136/bmjspcare-2011-000053.63

L H Tiew', V Drury², D Creedy ${ }^{1}$ Ministry of Health, National University of Singapore, Alice Lee Centre for Nursing Studies, Singapore; ${ }^{2}$ National University of Singapore, Singapore

Background Spiritual care is a central element of holistic and multidisciplinary care, but is not often integrated into practice. Some nurses may confuse spirituality with religion and refer this aspect of care to other professionals. There is little exploration of student nurses' perceptions and attitudes towards spirituality and spiritual care. Identifying student nurses' perceptions may inform educational content and practice development activities.

Purpose This exploratory study investigated nursing students' perceptions and attitudes about spirituality and spiritual care in practice.

Method In-depth interviews were conducted with 16 final year pre-registration nursing students from three different educational institutions offering a degree or diploma programme in Singapore. Data were analysed using the Miles and Huberman (1994) research method.

Results Three themes emerged in regards to the construct of spirituality: (1) being human, (2) spiritual well-being and (3) spiritual awareness. The construct of spiritual care revealed three themes: (1) antecedents for spiritual care, (2) role of nurses in spiritual care, and (3) nurses' spirituality. Themes relating to the construct of factors influencing spiritual care were: (1) personal factors; (2) system factors and (3) patient factors.

Conclusions Students perceived spirituality as an innate characteristic of individuals. Spiritual awareness was perceived to develop across the lifespan and was essential for spiritual well-being. Spiritual care required nurses to engage patients in meaningful ways. Cursory attention to spiritual care was considered ineffective and students perceived that a comprehensive spiritual assessment was important for care. Participants identified that nurses need to connect with patients in a unique spiritual care-giving relationship. Students also identified that nurses with certain attributes and spiritual awareness were more likely to provide spiritual care. However, students recognised that some nurses may not be equipped to address deeper levels of spiritual care. Education and professional development for effective spiritual care needs to be offered to nurses. 